\title{
I-131 MIBG Therapy for Advanced Stage III \& IV Neuroblastoma
}

\author{
Raef Riad $^{1 *}$, Magdy Kotb ${ }^{1}$, Walid Omar ${ }^{1}$, Ahmed Zaher ${ }^{1}$, Emad Ebied ${ }^{2}$, Alexander Gregory Pitman ${ }^{3}$, \\ Hussein Abdel Dayem ${ }^{4}$ \\ ${ }^{1}$ Nuclear Medicine Department, National Cancer Institute, Cairo University, Cairo, Egypt; ${ }^{2}$ Pediatric Oncology Department, National \\ Cancer Institute, Cairo University, Cairo, Egypt; ${ }^{3}$ Department of PET and Nuclear Medicine, Lake Imagimg, St. John of God Hospi- \\ tal, Victoria, Australia; ${ }^{4}$ Department of Radiology, New York Medical College, New York, USA. \\ Email: *raefriad@hotmail.com
}

Received June $23^{\text {rd }}$, 2011; revised July 19 ${ }^{\text {th }}$, 2011; accepted July 27 $7^{\text {th }}, 2011$.

\begin{abstract}
Radio-labelled MIBG therapy has been tried in the treatment of advanced stage III and IV neuroblastoma in an attempt to improve patients outcome. The use of radiolabelled MIBG to treat neuroblastoma has arisen from the high sensitivity and specificity of in vivo MIBG imaging for detection of primary and metastatic tumours. Although, clinical trials have been performed to determine the role of MIBG therapy, the overall therapeutic strategy of MIBG therapy is not precisely defined. Aim of the Work: to determine the impact of MIBG therapy on patients' outcome especially their quality of life. Patients \& Methods: 42 paediatric neuroblastoma patients are included in this study, 12 (28.5\%) stage III disease and 30 (71.5\%) stage IV. I-131 MIBG doses ranged from 100 to $150 \mathrm{mCi}$ with number of courses ranged from 1-8 according to response and toxicity. Results: Group I (stage III): 1/12 patients showed complete response, 5/12 patients had partial response and the other 6 patients remained stable. The 5 years event free survival (EFS) of stage III patients was $46.6 \%$ and the overall survival was $75 \%$. Group II (stage IV): 2/30 patients achieved CR, 9/30 patients showed PR to I-131 MIBG and the other 18 remained stable. The 5 years event free survival (EFS) of stage IV patients was $48.2 \%$ and the overall survival was 69\%. Conclusions: We conclude that 131I-MIBG therapy has favourable therapeutic effects that are translated into an overall improved outcome with good quality of life.
\end{abstract}

Keywords: I-131 MIBG, Neuroblastoma, Pediatrics

\section{Introduction}

Neuroblastoma is the most common solid extra cranial malignancy in children younger than 15 years. It accounts for $8 \%-10 \%$ of all childhood cancers and $15 \%$ of cancer deaths in children [1]. Although this tumor is chemo- and radio-sensitive, it is prone to relapse after initial induction of remission. Stage I and II tumours can be cured with surgery alone, whilst stage III tumours require preoperative chemotherapy. Sixty percent of neuroblastomas in children are diagnosed in stage IV, of whom many have biological markers of poor prognosis, such as MYCN amplification or 1p deletion [2]. One of the major goals of cancer treatment is to develop therapies affecting cancer cells while causing little or no damage to the normal counterparts. In this perspective numerous attempts have been made to bind anti-tumor compounds or radioactive isotopes to molecules specifically taken up by tumour cells. One example of these molecules is meta-iodobenzylguanidine (MIBG), a norepinephrine analogue which is taken up by organs rich in adrenergic innervation and/or catecholamine excretion. Therefore, I-131 radio-labelled MIBG allows successful imaging of these organs and neuroectodermally derived tumors, such as neuroblastomas, pheochromocytomas, paragangliomas, medullary thyroid carcinoma, carcinoid tumors and Merkel cell skin tumors. The use of radiolabelled MIBG to treat neuroectodermally derived tumors have arisen from the high sensitivity and specificity of in vivo MIBG imaging for detection of primary and metastatic tumours [3].

Radio-labelled MIBG therapy is most commonly used in the treatment of neuroblastoma, which is a high grade malignancy of childhood [3]. I-131MIBG was initially reserved for palliation of patients with recurrent disease. However, clinical trials evaluating the role of 131IMIBG as a first line drug, either as a single agent, or in combination with chemotherapy or myeloablation treat- 
ment, or in consolidation treatment have been performed with mixed results and significant potential side effects. The response rates varied between $20 \%$ and $60 \%$ in newly diagnosed and relapsed or refractory patients [4-9].

Despite all this information the precise role of 131IMIBG in the overall therapeutic strategy of neuroblastoma is far from being defined. In particular, it is still unclear whether I-131MIBG might improve the tumour response of patients who did not achieve complete remission with conventional therapy and are therefore predisposed to disease progression and death.

We conducted this retrospective study to present the experience of the National Cancer Institute, Cairo-Egypt with I-131MIBG therapy in neuroblastoma and to determine the impact of MIBG therapy on patients' outcome especially their quality of life. This study has been approved by the NCI ethics and research committee.

\section{Patients and Methods}

The study included 42 paediatric patients treated and followed up at the National Cancer Institute (NCI), Cairo University between March 1998 and Dec. 2008. All patients had a confirmed histopathology of neuroblastoma. Twelve patients (28.5\%) had stage III disease and 30 (71.5\%) were stage IV disease. Twenty five of the study patients (59.5\%) were males and 17 (40.5\%) were females. Their age ranged from 12 months to 11 years, with a mean of $4.8 \pm 3.1$ years. Frequencies of the disease primary sites were as follows: $29(69 \%)$ patients with supra-renal neuroblastoma, 7 (16.6\%) retro-peritoneal, 4 (16.6\%) para-vertebral and 2 (4.7\%) pre-sacral. Lymph node spread was seen in 9/12 patients of stage III disease. Bone deposits were detected in 26 patients. Hepatic deposits were present in 5 patients and brain metastasis was present in only one patient.

All the patients did I-131 MIBG total body scan before attempting I-131 MIBG therapy. All had residual tumour in the diagnostic scan at end of first-line therapy in both stage III and IV disease. Residual tumour was detected either at the primary site of the tumour and/or other metastatic lesions .

Further Eligibility criteria for I-131 MIBG therapy included normal CBC, liver and kidney functions.

\subsection{Patient Preparation before I-131 MIBG Therapy}

- Reviewing diagnostic investigations i.e. CT, MRI, I-131 MIBG diagnostic scan \& bone scan.

- $\quad$ CBC, liver function tests and kidney function tests were done one day before therapy.

- Measuring level of tumour markers (venyl mandilic acid VMA, homovanillic HVA in urine), serum neurone specific enolase NSE.

- Reviewing recent bone marrow aspiration or biopsy to exclude marrow infiltration.

- Thyroid gland uptake of radioiodine was blocked by oral administration of $2-3 \mathrm{mg} / \mathrm{kg} /$ day of Lugol's Iodine solution(saturated solution of KI 10\%) given for 3 days before and 7 days after 131I-MIBG infusion.

\subsection{I-131 MIBG Therapy}

The administered doses of I-131 MIBG ranged from 100 mCi for children weighing less than $20 \mathrm{~kg}$, up to 150 $\mathrm{mCi}$ for children weighing more than $20 \mathrm{~kg}$. Specific activity ranged from 1.4 to $2.3 \mathrm{GBq} \cdot \mathrm{mg}^{-1}$ (mean 1.8). I131 MIBG dose is injected in $100 \mathrm{ml}$ saline or $100 \mathrm{ml}$ $5 \%$ dextrose over 45 minutes. Close relatives actively participated in nursing care were provided with digital pocket dosimeter for radiation exposure monitoring after explaining radiation safety guidelines to them. Patients were discharged usually when the exposure rate at 1 meter is less than $30 \mathrm{mR} / \mathrm{hr}$. Once discharged they were checked weekly clinically and through laboratory tests for haematological indices, kidney \& liver functions to monitor any toxicity. Any Toxic effects attributable to I131 MIBG therapy were reported. The treatment was repeated every 4 - 6 weeks. Assessment of primary tumour and metastasis response was done after the third I131 MIBG therapy course unless evidence of progressive disease or toxic side effects had occurred.

In case of either disease improvement or stability after the third I-131 MIBG course, additional courses with an interval of 4 - 6 weeks between courses were administered.

\subsection{Evaluation of Tumour Response}

Tumour response was defined as follows:

- $\quad$ Complete response (CR), disappearance of primary tumour and of all metastatic lesions with normalization of urine catecholamines;

- Very good partial response (VGPR), $>90 \%$ volume reduction of the primary tumour with clearing of all measurable metastatic lesions with the exception of residual changes at skeletal scintigraphy, normalization of urine catecholamines;

- $\quad$ Partial response (PR), $>50 \%$ volume reduction of the primary tumour and of all measurable metastatic lesions,

- $\quad$ Stable disease (SD), $<50 \%$ reduction but $<25 \%$ increase in any existing lesion.

- $\quad$ Progressive disease (PD), increase $>25 \%$ of any measurable lesion or appearance of a new lesion [10].

Isolated decrease of I-131 MIBG uptake was not con- 
sidered evidence of tumour response. Response was evaluated as mentioned before 3 - 4 weeks following the third I-131 MIBG course by means of CT scan, and urinary catecholamines.

Quality of life: we intended to monitor changes in patients quality of life (him-herself \& relatives) in terms of degree of pain relief, ability of move and play and psychological burden.

\section{Results}

The 42 patients included in this study were arranged in two groups; Group I included 12 patients with stage III neuroblastoma, Group II included 30 patients with stage IV neuroblastoma.

\subsection{Group I}

Twelve patients out of the 42 patients included in this study were stage III disease All had partial tumour response to first-line treatment. Before I-131MIBG therapy 6 patients underwent surgery consisting of partial tumour resection in 3 patients, exploration \& biopsy in two patients and near total resection in the remaining patient with histological evidence of viable tumour cells in all surgical specimens.

Before starting I-131 MIBG all the patients showed elevated level of urinary catecholamine excretion \& pathological scintigraphic I-131 MIBG uptake at the site of the primary. Main patients' data are listed in Table 1.

Five patients received six I-131 MIBG courses, three patients received two courses and the remaining four patients: one received eight courses, one received 7 courses, one received five courses and one received two courses.

The interval between courses ranged from 4 to 6 weeks. One patient showed complete response (case no. 5) and is still alive disease free 132 month later. Five patients had partial response four of these five patients are alive with stable disease (cases no. 1, 6, 7, 9,) for 38 67 months (mean 50.5) (Figures 1(a)-(b)), and one patient (case no. 8) showed progressive disease and is alive for 21 months.

The remaining 6/12 patients remained with stable disease with I-131 MIBG therapy. Five patients out of these six showed progressive disease and three of them died (cases no. 2, 3, 4) and 2 are alive (cases no. 10, 12) for 20, 46 months. The remaining patient (case no. 11) remained stable and is alive for 46 months (Figures 2(a)-(b)).

Progressive disease was determined by appearance of new osseous deposits in I-131 MIBG scan, bone scan as well as increase in level of urinary catecholamine, while patients with stable disease showed no further progression in tumour by CT or in the I-131 MIBG scan and no increase in level of urinary catecholamines.

No further anti-tumour therapy was administered to all the patients of this group in conjunction with I-131 MIBG therapy.

The 5 year event free survival (EFS) of this group is $46.6 \%$ (Figure 3) and the overall survival is $75 \%$ with follow-up of 8 - 132 months (median, 38) (Figure 4).

\subsection{Group II}

Thirty patients out of the 42 patients included in this study were stage IV disease who achieved partial response with first-line therapy. Ten of them underwent partial surgical resection. Scintigraphy performed before I-131MIBG therapy was positive at the primary site and metastatic lesions \{bone deposits (26 patients), hepatic deposits (5 patients)\}. Main patient data are summarized

Table 1. Characteristics of group I (stage 3 patients).

\begin{tabular}{|c|c|c|c|c|c|c|c|}
\hline Case NO. & $\begin{array}{l}\text { Sex \& age } \\
\text { (years) }\end{array}$ & Primary tumor site & $\begin{array}{c}\text { Surgery after } 1^{\text {st }} \text { line } \\
\text { chemo }\end{array}$ & VMA \& HVA & $\begin{array}{l}\text { I-131 MIBG } \\
\text { therapy courses }\end{array}$ & $\begin{array}{l}\text { Response after } 3^{\text {rd }} \\
\text { MIBG course }\end{array}$ & $\begin{array}{l}\text { Clinical course and } \\
\text { outcome }\end{array}$ \\
\hline 1 & M, 4 & Rt. adrenal & Partial resection & elevated & 6 courses & PR & Alive 67 months, SD \\
\hline 2 & F, 3 & Lt. adrenal & exploration & elevated & 3 courses & SD & Dead 18 months, PD \\
\hline 3 & M, 3 & Rt. adrenal & exploration & elevated & 3 courses & SD & Dead 8 months, PD \\
\hline 4 & M, 1 & Rt. adrenal & No & elevated & 2 courses & $\mathrm{SD}$ & Dead 12 months, PD \\
\hline 5 & F, 2.5 & Pre-sacral & Near total & elevated & 8 courses & CR & Alive 132 months,CR \\
\hline 6 & M, 1 & Rt. adrenal & No & elevated & 6 courses & PR & Alive 55 months, SD \\
\hline 7 & M, 1.5 & Retro-peritoneal & No & elevated & 6 courses & PR & Alive 42 months, SD \\
\hline 8 & F, 2.5 & Lt. adrenal & No & elevated & 5 courses & PR & Alive 21 months, $\mathrm{PD}$ \\
\hline 9 & M, 12 & Pre-sacral & Partial resection & elevated & 6 courses & PR & Alive 38 months, SD \\
\hline 10 & F, 2.5 & Lt. adrenal & Partial resection & elevated & 6 courses & SD & Alive 46 months, PD \\
\hline 12 & $\mathrm{~F}, 4$ & Retro-peritoneal & No & elevated & 3 courses & SD & Alive 20 months, PD \\
\hline
\end{tabular}

$\mathrm{CR}$, complete remission; $\mathrm{PR}$, partial remission; SD, stable disease; $\mathrm{PD}$, progressive disease. 


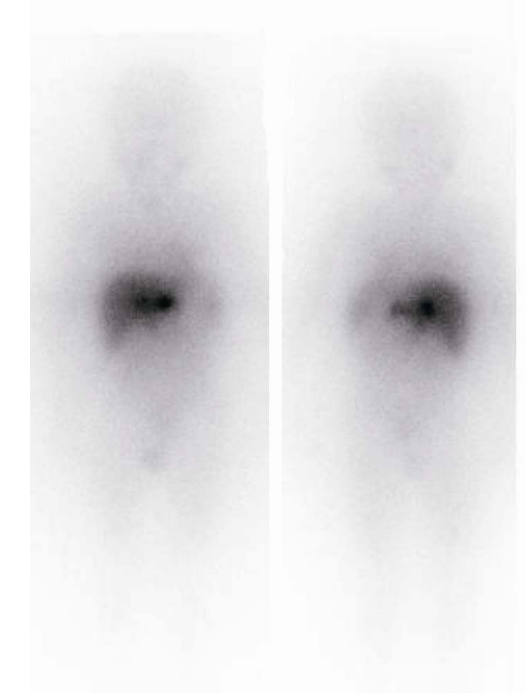

(a)

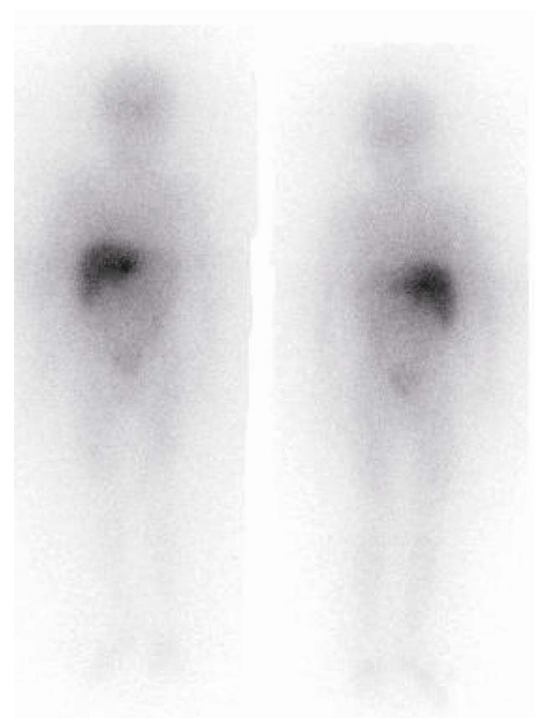

(b)

Figure 1. (a) Pre-MIBG therapy scan anterior (left) and posterior (right) images showing retro-peritoneal neuroblastoma. (b) Post MIBG therapy scan anterior (left) and posterior (right) images showing very good partial response in the form of more than $80 \%$ clearance of the primary mass (Case 7 of group I patients).

\section{in Table 2.}

Two patients received one I-131MIBG course of therapy, 4 received twocourses, 13 received three courses, one received four courses, two received five courses, six received six courses and two received seven courses. Intervals between courses as mentioned ranged from 4 to 6 weeks.

Two patients (cases no. 18,19) achieved CR (clearing of the primary tumour and two bone lesions) and are still alive disease-free 64 and 69 months later.

Nine patients showed PR to I-131 MIBG therapy involving the primary tumour (only the primary tumour)in five cases (cases no. 1, 8, 12, 23, 29), together with bone lesions in 3 patients(cases no. 4, 14, 20) and together with hepatic lesion in one patient (case no. 17). All the nine patients are alive at 16 - 57months (mean 30.6) among whom seven are alive with stable disease (cases no. $4,12,14,17,20,23,29)$ and two patients are alive with progressive disease (cases no. 1, 8).

Eighteen patients remained stable after I-131 MIBG therapy. Among them six are alive with PD (cases no. 2, $5,7,11,13,15)$ at 10 - 23months (mean 18.3) and four are alive with stable disease (cases no. 3, 16, 21, 30) at 17 - 25 months (mean 26.5), while the remaining eight patients died.

The last patient (case no. 22) developed PD and died within 15 months (Figures 5(a)-(b)).

The 5-year EFS of this group is $48.2 \%$ (Figure 6) and overall survival is 69\% (Figure 7) with follow up period of 8 - 69 months. (median 22).

Nearly the same results are achieved in the 2 groups under study (stage III and stage IV disease). This may be attributed to the fact that stage III disease patients are already advanced from the start, also may be because we used the same small doses of MIBG for both groups. May be it would had been a good idea to give larger doses related to the tumour burden. However this approach might have increased the toxicity.

We noticed marked improvement in pain in 22 patients out of the 26 patients with bone metastasis (84.5\%). Thirty two out of the 42 patients (76\%) included in this study showed better quality of life in the form of pain relief, enjoying longer time of playing without treatment burden , enjoying life with no toxic effects of therapy as well as partially relieving the psychological burden of the children and their parents. CR, complete remission; $\mathrm{PR}$, partial remission; SD, stable disease; PD, progressive disease.

\section{Discussion}

Long-term survival of children with inoperable or disseminated neuroblastoma diagnosed after the age of one year remains largely unsatisfactory mainly because chemotherapy treatment usually fails to eradicate the disease [11-13]. Thus, alternative therapies are being trialled for the benefit of increasing long term survival and improving quality of life. Radiolabelled benzylguanidine with Iodine 131 has been used in the treatment of neural crest tumours and seemed suitable due to it's physical characteristics. The radiation emitted from I-131 proved to be effective to induce damage of large tumour lesions [14]. 


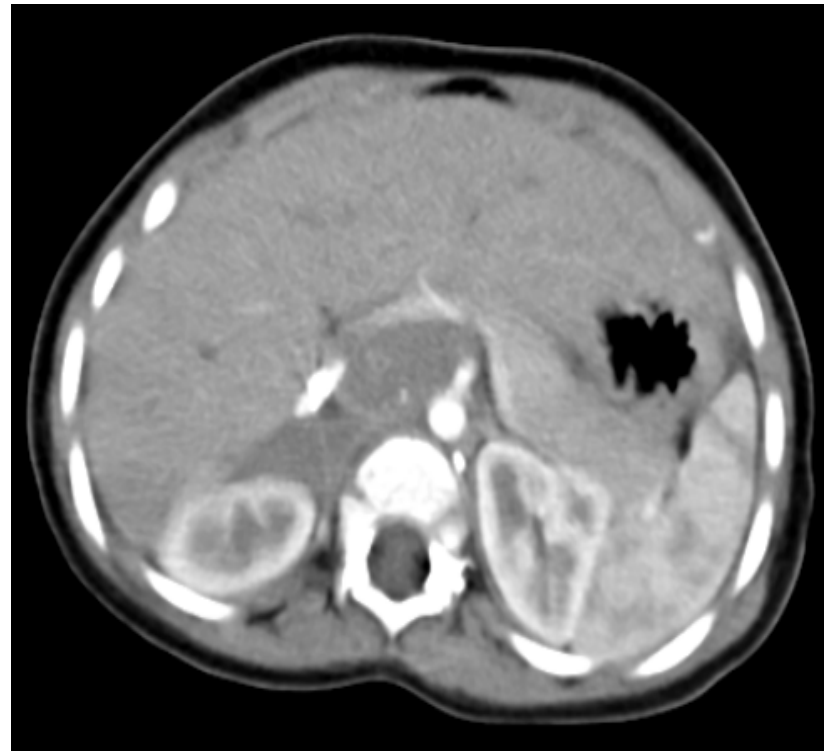

(a)

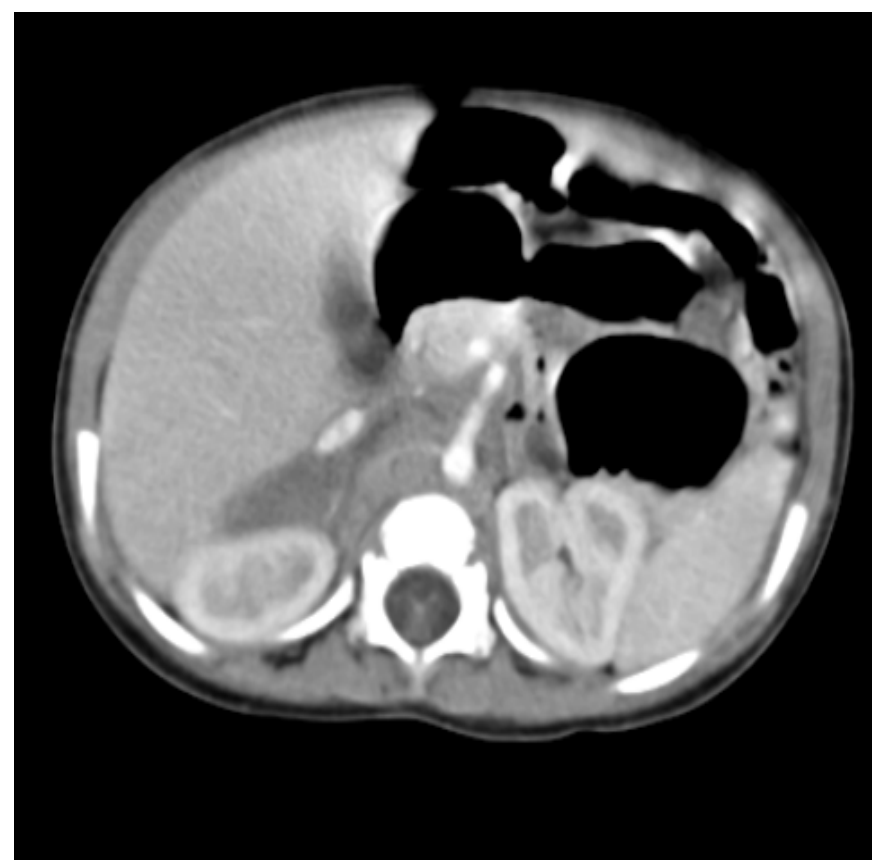

(b)

Figure 2. (a) Pre-MIBG therapy scan (left) and CT cut (right) showing Rt. Supra-renal mass. (b) Post therapy MIBG scan (left) and CT cut (right) showing no change in size and activity of the Rt. Supra-renal mass after 7 courses of MIBG therapy (Case 11 of group I patients). 


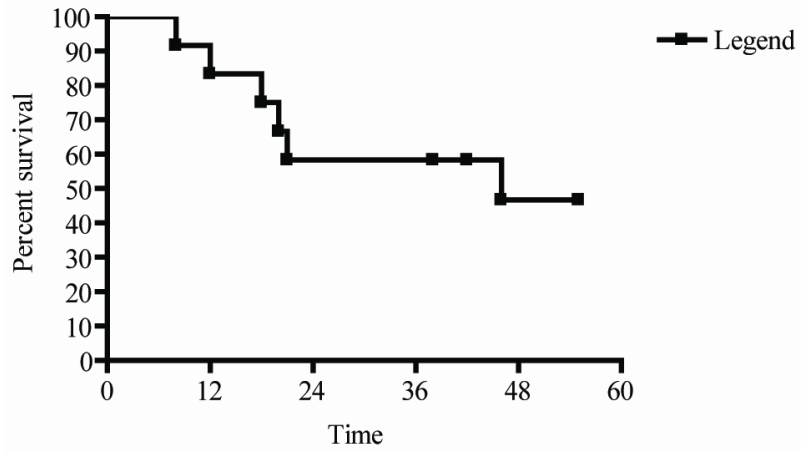

Figure 3. Five years event free survival of stage 3 patients.

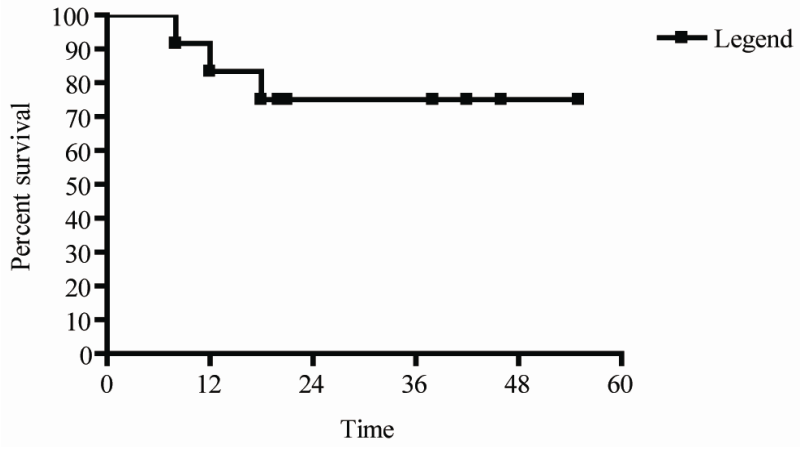

Figure 4. Overall survival of stage 3 patients.

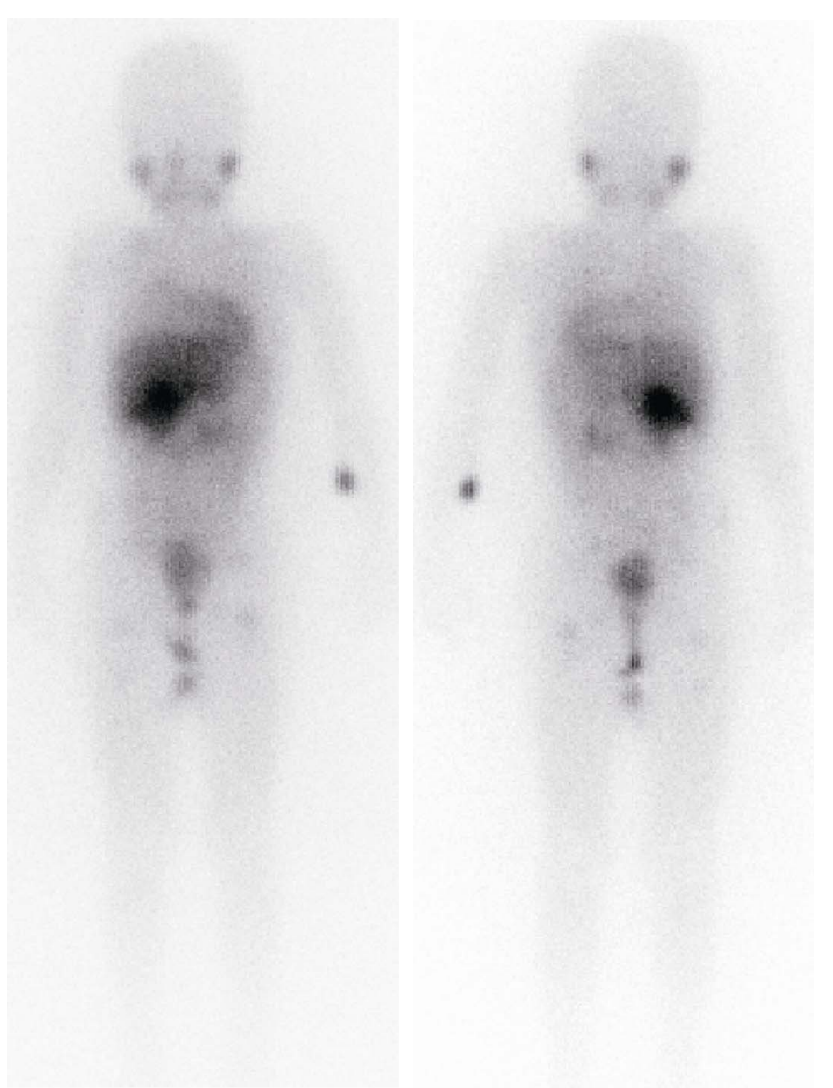

(a)

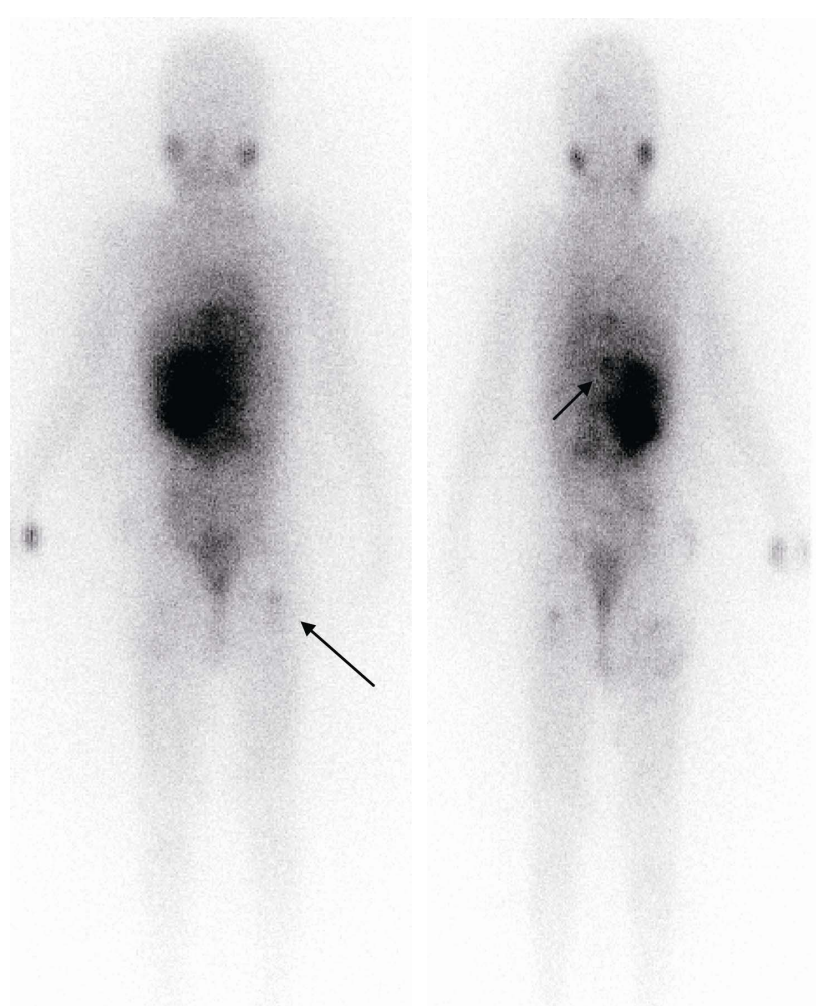

(b)

Figure 5. (a) Pre-MIBG therapy scan anterior (left) and posterior (right) images showing Rt. Supra-renal neuroblastoma. (b) Post MIBG therapy scan anterior (left) and posterior (right) images showing progression of the disease in the form of increase in the primary lesion and appearance of new MIBG avid mid dorsal and Lt. Upper femoral lesions (arrowed) (Case 22).

I-131 MIBG therapy for neuroblastoma has been used since the late 1980 and demonstrated objective tumour responses in $20 \%-60 \%$ of patients failing to respond to chemotherapy treatment protocols [15-17]. In addition, significant pain relief was noticed even in patients who did not exhibit objective responses [18]. Similarly in the present study, we noticed marked improvement in pain in 22 (84.5\%) patients out of the 26 patients with bone me- tastasis. Thirty two patients out of the 42 (76\%) patients included in this study showed better quality of life in the form of pain relief, enjoying longer time of playing without treatment burden, enjoying life with no toxic effects of therapy as well as partially relieving the psychological burden of the children and their parents.

Lastly, attempts are being made to evaluate whether I131 MIBG given as front-line therapy may improve the 
Table 2. Characteristics of stage 4 patients.

\begin{tabular}{|c|c|c|c|c|c|c|c|}
\hline Case NO. & $\begin{array}{l}\text { Sex \& age } \\
\text { (years) }\end{array}$ & Primary tumor site & $\begin{array}{c}\text { Surgery after } 1^{\text {st }} \text { line } \\
\text { chemo }\end{array}$ & VMA \& HVA & $\begin{array}{l}\text { I-131 MIBG } \\
\text { therapy courses }\end{array}$ & $\begin{array}{l}\text { Response after } 3^{\text {rd }} \\
\text { MIBG course }\end{array}$ & $\begin{array}{c}\text { Clinical course and } \\
\text { outcome }\end{array}$ \\
\hline 1 & M, 11 & Lt. adrenal & No & Elevated & 4 courses & PR & Alive 23 months PD \\
\hline 2 & M, 4 & Retro-peritoneal & No & Elevated & 3 courses & SD & Alive 22 months PD \\
\hline 3 & F, 8 & Para-vertebral & Partial resection & Elevated & 3 courses & SD & Alive 25 months SD \\
\hline 4 & F, 2 & Retro-peritoneal & No & Elevated & 6 courses & PR & Alive 31 months SD \\
\hline 5 & $\mathrm{M}, 10$ & Rt. adrenal & No & Elevated & 2 courses & SD & Alive 23 months PD \\
\hline 6 & M, 2 & Lt. adrenal & No & Elevated & 1 course & SD & Dead 12 months PD \\
\hline 7 & M, 4 & Rt. adrenal & No & Elevated & 3 courses & SD & Alive 19 months PD \\
\hline 8 & M, 9 & Rt. adrenal & No & Elevated & 5 courses & PR & Alive 18 months PD \\
\hline 9 & M, 11 & Rt. adrenal & No & Elevated & 3 courses & SD & Dead 13 months PD \\
\hline 10 & M, 9 & Para-vertebral & No & Elevated & 2 courses & SD & Dead 11 months PD \\
\hline 11 & $\mathrm{~F}, 4.5$ & Rt. adrenal & No & Elevated & 3 courses & SD & Alive 19 months PD \\
\hline 12 & M, 2 & Lt. adrenal & Partial resection & Elevated & 6 courses & PR & Alive 16 months SD \\
\hline 13 & F, 6 & Lt. adrenal & No & Elevated & 3 courses & SD & Alive 17 months PD \\
\hline 14 & F, 5 & Lt. adrenal & Partial resection & Elevated & 5 courses & PR & Alive 28 months SD \\
\hline 15 & M, 8 & Para-vertebral & No & Elevated & 3 courses & SD & Alive 10 months PD \\
\hline 16 & F, 4 & Retro-peritoneal & No & Elevated & 3 courses & SD & Alive 40 months SD \\
\hline 17 & F, 5 & Rt. adrenal & Partial resection & Elevated & 6 courses & PR & Alive 42 months SD \\
\hline 18 & F, 4 & Lt. supra-renal & Partial resection & Elevated & 7 courses & CR & Alive 64 months CR \\
\hline 19 & F, 2 & Rt. adrenal & Partial resection & Elevated & 7 courses & CR & Alive 69 months CR \\
\hline 20 & M, 3 & Lt. adrenal & Partial resection & Elevated & 6 courses & PR & Alive 57 months SD \\
\hline 21 & M, 3.5 & Lt. adrenal & No & Elevated & 3 courses & SD & Alive 24 months SD \\
\hline 22 & M, 4 & Rt. adrenal & Partial resection & Elevated & 2 courses & $\mathrm{PD}$ & Dead 15 months PD \\
\hline 23 & M, 11 & Rt. adrenal & Partial resection & Elevated & 6 courses & PR & Alive 37 months SD \\
\hline 24 & M, 3 & Lt. adrenal & No & Elevated & 1 course & SD & Dead 8 months PD \\
\hline 25 & F, 3 & Retro-peritoneal & No & Elevated & 2 courses & SD & Dead 13 months PD \\
\hline 26 & F, 3 & Lt. adrenal & No & Elevated & 3 courses & SD & Dead 15 months PD \\
\hline 27 & M, 11 & Lt. adrenal & No & Elevated & 3 courses & SD & Dead 9 months PD \\
\hline 28 & M, 3 & Para-vertebral & No & Elevated & 3 courses & SD & Dead 11 months PD \\
\hline 29 & M, 9 & Retro-peritoneal & Partial resection & Elevated & 6 courses & PR & Alive 24 months SD \\
\hline 30 & F, 5 & Rt. adrenal & No & Elevated & 3 courses & SD & Alive 17 months SD \\
\hline
\end{tabular}

$\mathrm{CR}$, complete remission; $\mathrm{PR}$, partial remission; SD, stable disease; $\mathrm{PD}$, progressive disease.

outcome of these patients without causing excessive toxicity $[18,19]$. To our knowledge, no prospective study has been carried out so far to evaluate the true potential value of I-131MIBG therapy and to identify the group of patients who may profit best from this treatment modality. The difficulties in design of such studies include the high cost of the drug, many centres do not possess the facilities to deliver this treatment to young patients, and the burden of keeping patient in a radiation protected environment with limited parental contact extended for few days.

Garaventa et al., 1991, studied 31 patients treated with I-131MIBG after disease relapse or progression. They reported that the rate and degree of responses were lower in the presence of bulky disease, a high number of MIBG-positive lesions, long duration of previous therapy and overt bone marrow infiltration [11].

On 1999, Garaventa et al., published their work on MIBG therapy in children with high-risk neuroblastoma who responded to first-line therapy without achieving CR, 


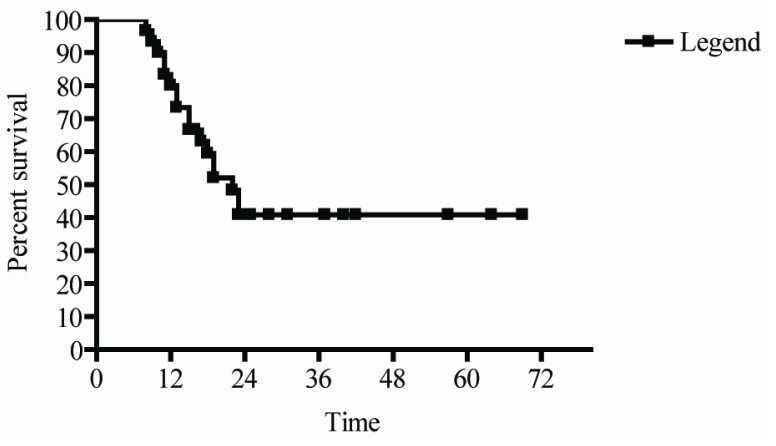

Figure 6. Five years event free survival of stage 4 patients.

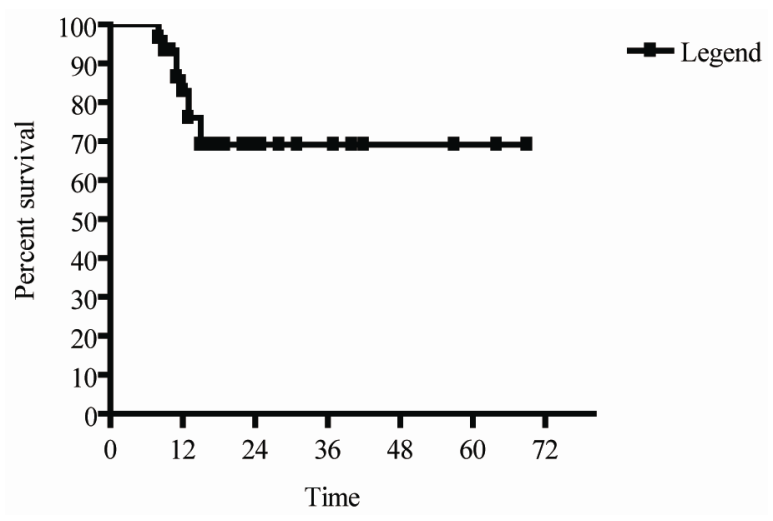

Figure 7. Overall survival of stage 4 patients.

provided that the residual primary tumour and/or metastases were clearly MIBG avid. They reported that MIBG therapy showed very good partial response in 4/13 patients with stage III neuroblastoma, 1 patient showed disease progression and 8 patients showed stable disease. Regarding stage IV patients, 1/30 patients showed CR, 10 patients showed $\mathrm{PR}, 15$ patients had no response to MIBG therapy and 4 patients showed progressive disease [20]. They stated the 5-year event-free survival (EFS) of stage III neuroblastoma to be $92 \%( \pm 0.07)$ and that of stage IV to be $40 \%( \pm 0.08)$ [20].

In the present study, our results indicate that patients with stage III disease did benefit from MIBG therapy. One patient showed complete response and is still alive disease free 132 month later, five patients had partial response, four of them are alive, and the remaining 6 patients remained stable with three of them alive. The 5 years event free survival (EFS) of stage 3 patients was $46.6 \%$ and the overall survival was $75 \%$.

Regarding stage IV patients, two patients achieved CR and are still alive disease-free 64 and 69 months later. Nine patients showed PR to I-131 MIBG, all the nine patients are alive at 16 - 57 months (mean 30.6) among whom seven are alive with stable disease and two patients are alive with progressive disease. Eighteen pa- tients remained stable after I-131 MIBG therapy, among them six are alive with PD and four are alive with stable disease, while the remaining eight patients died. The last patient developed PD and died within 15 months. The 5 years event free survival (EFS) of stage IV patients was $48.2 \%$ and the overall survival was $69 \%$.

Our results are different from Garaventa et al., 1999 concerning stage III neuroblastoma, this may be attributed to the fact that stage III disease patients are already advanced from the start, also may be because we used the same doses of MIBG for both groups [20].

\section{Conclusions}

We conclude that I-131 MIBG therapy has favourable therapeutic effects that are translated into an overall improved outcome with good quality of life. To our opinion improving quality of life is one of great achievement of I-131 MIBG therapy.

Controlled clinical trials should be considered to evaluate the true potential of I-131 MIBG therapy.

\section{REFERENCES}

[1] G. M. Brodeur and J. M. Maris, “Neuroblastoma,” In: P. A. Pizzo and D. G. Poplack, Eds., Principles and Practice of Pediatric Oncology, Lippincott Williams \& Wilkins, Philadelphia, 2006, pp. 933-970.

[2] K. Oberg, "Advances in Chemotherapy and Biotherapy of Endocrine Tumors," Current Opinion in Oncology, Vol. 10, No. 1, 1998, pp. 58-65. doi:10.1097/00001622-199801000-00010

[3] W. J. Oyen, L. Bodei, F. Giammarile, H. R. Maecke, J. Tennvall, M. Luster and B. Brans, "Targeted Therapy in Nuclear Medicine-Current Status and Future Prospects," Annals of Oncology, Vol. 18, No. 11, 2007, pp. 17821792. doi:10.1093/annonc/mdm111

[4] M. Miano, A. Garaventa, et al., "Megatherapy Combining I(131) Metaiodobenzylguanidine and High-Dose Chemotherapy with Haematopoietic Progenitor Cell Rescue for Neuroblastoma," Bone Marrow Transplant, Vol. 27, 2001, pp. 571-574.

[5] J. De Kraker, C. A. Hoefnagel, H. Caron, et al., "First Line Targeted Radiotherapy, a New Concept in the Treatment of Advanced Stage Neuroblastoma," European Journal of Cancer, Vol. 31, No. 4, 1995, pp. 600-602. doi:10.1016/0959-8049(95)00063-O

[6] S. S. Goldberg, K. DeSantes, J. P. Huberty, et al., "Engraftment after Myeloablative Doses of 131I-Metaiodobenzylguanidine Followed by Autologous Bone Marrow Transplantation for Treatment of Refractory Neuroblastoma," Medical and Pediatric Oncology, Vol. 30, No. 6, 1998, pp. 339-346.

doi:10.1002/(SICI)1096-911X(199806)30:6<339::AID-M PO7>3.0.CO;2-F

[7] C. A. Hoefnagel, P. A. Voute, J. de Kraker and H. R. Marcuse, "Radionuclide Diagnosis and Therapy of Neural 
Crest Tumors Using Iodine-131 Metaiodobenzylguanidine,” Journal of Nuclear Medicine, Vol. 28, 1987, pp. 308-314.

[8] C. A. Hoefnagel, J. De Kraker, R. A. Valdes Olmos and P. A. Voute, "131I-MIBG as a First-Line Treatment in HighRisk Neuroblastoma Patients,” Nuclear Medicine Communications, Vol. 15, No. 9, 1994, pp. 712-717. doi:10.1097/00006231-199409000-00008

[9] S. Meller, "Targeted Radiotherapy for Neuroblastoma," Archives of Disease in Childhood, Vol. 77, 1997, pp. 389391. doi:10.1136/adc.77.5.389

[10] G. M. Brodeur, J. Pritchard, F. Berthold, et al., "Revisions of the International Criteria for Neuroblastoma Diagnosis, Staging and Response to Treatment," Journal of Clinical Oncology, Vol. 11, 1993, pp. 1466-1477.

[11] A. Garaventa, B. De Bernardi, C. Pianca, et al., "Localized but Unresectable Neuroblastoma: Treatment and Outcome of 145 Cases,” Journal of Clinical Oncology, Vol. 9, 1993, pp. 1770-1779.

[12] G. M. Haase, J. B. Atkinson, D. O. Stram, J. N. Lukens and K. K. Matthay, "Surgical Management and Outcome of Locoregional Neuroblastoma: Comparison of the Children Cancer Group and the International Staging System,” Journal of Pediatric Surgery, Vol. 30, No. 2, 1995, pp. 289-295. doi:10.1016/0022-3468(95)90576-6

[13] T. Philip, R. Ladenstein, C. Lasset, et al., "1070 Myeloablative Megatherapy Procedures Followed by Stem Cell Rescue for Neuroblastoma: 17 Years of European Experience and Conclusions," European Journal of Cancer, Vol. 33, No. 12, 1997, pp. 2130-2135. doi:10.1016/S0959-8049(97)00324-9

[14] W. Weber, J. Weber and R. Senekowitsch-Schmidtke, “Therapeutic Effect of M-[I-131]- and M-[I-125] Iodo- benzylguanidine on Neuroblastoma Multicellular Tumor Sphe- roids of Different Sizes,” Cancer Research, Vol. 56, 1996, pp. 5428-5434.

[15] D. Schwabe, St. Sahm, V. Gerein, et al., “131-Metaiodobenzylguanidine Therapy of Neuroblastoma in Childhood,” European Journal of Pediatrics, Vol. 146, No. 3, 1987, pp. 246-250. doi:10.1007/BF00716467

[16] T. Klingebiel, J. Treuner, G. Ehninger, et al., “[I-131]Metaiodobenzylguanidine in the Treatment of Metastatic Neuroblastoma," Cancer Chemotherapy and Pharmacology, Vol. 25, No. 2, 1989, pp. 143-148. doi:10.1007/BF00692356

[17] L. S. Lashford, I. J. Lewis, S. L. Fielding, et al., "Phase I/II Study of Iodine 131 Metaiodobenzylguanidine in Chemoresistant Neuroblastoma: A United Kingdom Children Cancer Study Group Investigation,” Journal of Clinical Oncology, Vol. 10, 1992, pp. 1889-1896.

[18] E. J. Van Hasselt, H. A. Heij, J. De Kraker, A. Vos and P. A. Voûte, "Pretreatment with [I-131]Metaiodobenzylguanidine and Surgical Resection of Advanced Neuroblastom,” European Journal of Pediatric Surgery, Vol. 6, No. 3, 1996, pp. 155-159. doi:10.1055/s-2008-1066495

[19] R. Mastrangelo, A. Tornesello and S. Mastrangelo, "Role of 131 Metaiodobenzylguanidine in the Treatment of Neuroblastoma," Medical and Pediatric Oncology, Vol. 31, No. 1, 1998, pp. 22-26. doi:10.1002/(SICI)1096-911X(199807)31:1<22::AID-MP O5>3.0.CO;2-1

[20] A. Garaventa, O. Bellagamba, M. S. Lo Piccolo, et al., "I-131 Metaiodobenzylguanidine (I-131MIBG) Therapy for Residual Neuroblastoma: A Mono-Institutional Experience with 43 Patients," British Journal of Cancer, Vol. 81, No. 8, 1999, pp. 1378-1884. 